\title{
Protocol
}

\section{A prospective study to evaluate pelvic bone mineral density changes in patients of cervical cancer undergoing chemoradiotherapy: study protocol}

\author{
Divyesh Kumar ${ }^{1}$, Tulika Singh ${ }^{2}$, Pooja Bansal $^{3}$, Bhavana Rai ${ }^{1}$
}

\begin{abstract}
${ }^{1}$ Department of Radiotherapy and Oncology, ${ }^{2}$ Department of Radiodiagnosis, ${ }^{3}$ Department of Biostatistics, Post Graduate Institute of Medical Education and Research, Chandigarh, India
\end{abstract}

Received: 21 July 2019

Revised: 06 August 2019

Accepted: 07 August 2019

\author{
*Correspondence: \\ Dr. Divyesh Kumar, \\ E-mail: divyeshanand1@gmail.com
}

Copyright: () the author(s), publisher and licensee Medip Academy. This is an open-access article distributed under the terms of the Creative Commons Attribution Non-Commercial License, which permits unrestricted non-commercial use, distribution, and reproduction in any medium, provided the original work is properly cited.

\begin{abstract}
Background: Cancer treatment induced bone loss has been retrospectively studied as a distinct entity in gynaecological cancers. Amongst gynaecological cancers, cervical cancer is the leading cause of mortality and morbidity, majorly in developing countries. Concurrent chemoradiation (CCRT) is considered as the standard of care in managing these patients. Persistent low back ache is often reported as a potential post treatment sequalae by long term survivors of cervical malignancy. Various retrospective studies done have observed reduced density and osteopenia of the bones in the irradiated area, as a possible etiologic factor for persistent low back ache.

Methods: We in this prospective clinical trial propose to prospectively and systematically evaluate the changes in pelvic bone density in patients of cervical cancer receiving chemoradiotherapy using dual energy X-ray absorptiometry (DEXA) scan done pre and post treatment, and correlate the changes with occurrence and severity of persistent low back ache. Low back ache will be evaluated using Oswestry low back pain disability scale, scoring for which shall be done pre-treatment and then at post treatment at 2 monthly interval for 1 year on follow up.

Conclusions: Results from the trial might bring-forth the changes in the density of pelvic bones in patients of cancer cervix undergoing concurrent chemoradiation and its correlation with low back ache, if any.

Trial Registration: This trial is registered with number CTRI/2017/05/008606.
\end{abstract}

Keywords: Cancer cervix, Chemoradiotherapy, Pelvic bone

\section{INTRODUCTION}

Cervical cancer is one of the leading causes of morbidity and mortality amongst the gynecological cancers worldwide. ${ }^{1}$ The South Asian region harbors one fourth of the burden of cervical cancer. ${ }^{2}$ Concurrent chemo radiotherapy (CRT) is the standard of care for locally advanced cervical cancer. Being a curable disease with expected long term survival rates ranging from $60 \%$ to $80 \%$, reduction of treatment related long term toxicities and improvement of quality of life assume greater importance in these patients. Among various post treatment morbidities, persistent low back ache is often reported by the long term survivors, but seldom studied systematically. Various retrospective studies have observed reduced density and osteopenia of the bones in the irradiated area. ${ }^{3-5}$

Dual energy X-ray absorptiometry (DEXA) is an enhanced form of X-ray imaging, and is one of the established methods for quantifying loss of bone mineral density (BMD). Bone densitometry with DEXA has been 
found to be more accurate than DPA, and it has been extensively developed to replace DPA and used to evaluate BMD. ${ }^{6}$ In this study, we propose to measure the BMD of lower lumbar vertebrae (L5) and bilateral hips, which receive significant doses of radiation during treatment of cervical cancer prior to start of chemoradiotherapy and again after 12 months of irradiation, the DEXA imaging will be repeated, to quantify the changes in BMD. Since pelvic irradiation with curative doses for treatment of cervical cancer invariably results in permanent ovarian failure, which might also result in generalised osteopenia, changes in density of non-irradiated bones like dorsal vertebrae (D12) are simultaneously measured, to serve as a control.

Low back pain (LBP) is a non-traumatic musculoskeletal disorder affecting the lower back. ${ }^{7}$ Since low back ache is a frequent symptom at the time of diagnosis of cervical cancer, and it is subjective in nature, Oswestry low back pain disability scale, which can objectively quantify the disability due to persistent low back ache, will be employed.

The purpose of our study is to prospectively analyse the changes in bone density of the pelvic bones in the irradiated area, in patients of cervical cancer treated with CRT, and to correlate these changes with occurrence and severity of persistent low back ache.

\section{Aims and objectives}

- To assess the pelvic bone density changes in patients of cervical cancer undergoing chemoradiotherapy.

- To correlate the changes in bone density with occurrence and severity of persistent low back ache.

\section{METHODS}

This is a prospective, observational, nonrandomised study being conducted in department of Radiotherapy, PGIMER, Chandigarh. This study is an ongoing clinical trial. Total of 106 patients are planned to be enrolled over a period of 2 years. Work flow pattern as depicted in Figure 1.

\section{Inclusion criteria}

Inclusion criteria were histological diagnosis of cervical cancer; FIGO stage IB to IVA; good performance status, suitable for curative chemoradiotherapy; consenting for participation and follow up.

\section{Exclusion criteria}

Exclusion criteria were operated cases of cervical cancer; previous history of pelvic irradiation; history of pelvic trauma; patients on hormone replacement or long term corticosteroids; pelvic bone metastasis.

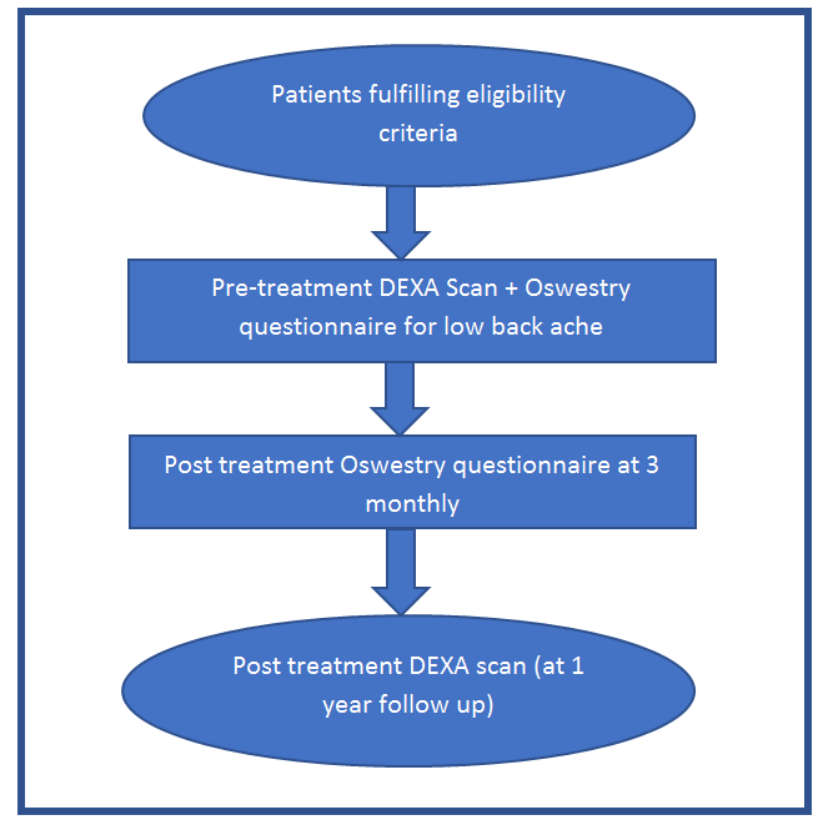

Figure 1: Workflow pattern.

\section{Investigations and work up}

All patients will undergo:

- History and physical examination.

- Cervical biopsy for histological diagnosis.

- Chest X ray.

- Complete hemogram.

- Renal function tests.

- Serum electrolytes.

- DEXA scan.

- Filling of Oswestry low back ache questionnaire.

\section{Procedure}

In this study each patient will undergo DEXA imaging before start of chemoradiotherapy treatment and at 1 year of completion of treatment, where bone mineral density of bilateral femoral heads, L5 and D12 vertebrae will be measured-score will be calculated for all three mentioned sites and will be subsequently analysed statistically.

Low back pain score will be evaluated using the Oswestry scale in which all patients will be asked to fill a 10-point questionnaire before initiation of treatment and at each 2 monthly follow up for 1 year, post completion of chemoradiation. Changes in the disability scores will be correlated with bone mineral density changes using appropriate statistical tests.

All patients will be treated by standard departmental treatment protocol for radiotherapy, using computed tomography based planning in which each patient will receive external radiation of 46 Gy in 23 fractions, over 4.5 weeks, concurrently with weekly cisplatinum at 40 
$\mathrm{mg} / \mathrm{m}^{2}$, followed by a brachytherapy boost of $9 \mathrm{~Gy}$ delivered twice at weekly interval.

\section{Follow up}

After treatment completion all patients will be closely followed up once every two months in the first year. All patients will receive oral calcium and vitamin supplements. At each follow up, apart from physical examination, low back pain score measurements will be documented using Oswestry scale. After 12 months post treatment, DEXA imaging will be repeated for bone density analysis.

\section{Statistical analysis}

Considering the retrospective reports observing a change in mean bone density $\mathrm{Z}$ scores of at least 0.67 , a sample size of 96 patients would be necessary with an alpha error of 0.05 and power of $90 \%$. Assuming a dropout rate of $10 \%, 107$ patients need to be accrued in this study. Estimated duration of the study is 2 years. Appropriate statistical tests will be done using SPSS version 20. Apart from descriptive statistics, changes in mean bone density before and after treatment will be analysed using paired $t$ test. Changes in low back ache scores over the first year of treatment will be analysed using Wilcoxon's matched pairs rank test. Univariate and Multivariate analyses will be used to identify correlation between changes in bone mineral density and occurrence and severity of persistent low back ache. A p $<0.05$ will be considered for statistical significance.

\section{DISCUSSION}

Cervical cancer is the commonest cancer cause of death among women in developing countries. ${ }^{8}$ Most of the patients of cervical cancer in India present with a locally advanced disease. 9 For these patients, concurrent chemoradiotherapy followed by brachytherapy is the standard of care, and offers overall survival rates of $60 \%$ to $80 \% .^{10}$

Various studies have focussed on analysing delayed toxicity in the form of genitourinary, gastrointestinal and psychosocial complications. ${ }^{11}$ However, multiple other factors including persistent low back ache contribute to decreased quality of life in long term survivors of cervical cancer. $^{12,13}$

Cancer treatment induced bone loss has been studied as a distinct entity in gynaecological cancers. ${ }^{14}$ Various reports have observed increased susceptibility to fractures and bone loss in patients with cancer treatment induced osteoporosis, leading to significant impairment of quality of life. ${ }^{15}$ Park et al have considered pelvic radiotherapy to be a significant risk factor for sacral insufficiency fractures in their study. ${ }^{16}$ Baxter et al reported a 5 year cumulative occurrence rate of radiation related insufficiency fractures in approximately $8.2 \%{ }^{17}$
Multiple studies have observed a causal association between therapeutic doses of radiation and decrease in bone mineral density in gynaecological cancers. ${ }^{18-20}$ Okonogi et al have reported significant reduction in mean bone mineral densities within one year of pelvic radiotherapy in both pre-menopausal and postmenopausal women. ${ }^{21}$ Most of these studies have used DEXA as the imaging modality for identifying changes in bone mineral density. It is considered as one of the most accurate modalities for diagnosis of osteopenia. ${ }^{22}$

In spite of multiple studies independently reporting decrease in bone density changes, increase in osteoporotic fractures, and decrease in quality of life due to persistent low back ache, there have been no prospective studies reporting a significant correlation between bone density changes and persistent low back ache. Being a subjective entity, quantification of low back ache requires special tools. Oswestry low back pain disability scale is one such questionnaire, which is considered as a gold standard for quantifying low back ache related disability. ${ }^{23}$

In our study, we propose to prospectively and systematically evaluate the changes in pelvic bone density in patients of cervical cancer receiving chemoradiotherapy, and correlate the changes with occurrence and severity of persistent low back ache.

Funding: No funding sources

Conflict of interest: None declared

Ethical approval: The study was approved by the Institutional Ethics Committee (NK/2721/Study/407)

\section{REFERENCES}

1. Sankaranarayanan R, Ferlay J. Worldwide burden of gynaecological cancer: the size of the problem. Best Pract Res Clin Obstet Gynaecol. 2006;20:207-25.

2. Laikangbam $P$, Sengupta S, Bhattacharya $P$, Duttagupta C, Dhabali Singh T, Verma Y, et al. A comparative profile of the prevalence and age distribution of human papillomavirus type 16/18 infections among three states of India with focus on northeast India. Int J Gynecol Cancer. 2007; 17:10717.

3. Theresa AG. Bone Loss and Fracture Risk Associated with Cancer Therapy. The Oncologist. 2006;11(10):1121-31.

4. Oh YL, Yoon MS, Suh DS, Kim A, Kim MJ, Lee JY, et al. Changes in bone density after cancer treatment in patients with cervical and endometrial cancer. J Cancer. 2015;6(1):82-9.

5. Hopewell JW. Radiation-therapy effects on bone density. Med Pediatr Oncol. 2003;41(3):208-11.

6. Sartoris DJ, Resnick D. Dual-energy radiographic absorptiometry for bone densitometry: current status and perspective. Am J Roentgenol. 1989;152:241-6.

7. Bisoi S, Mondal T, Haldar D. Low Back Pain And Disability: A Cross Sectional Study Among Patients 
Attending Outpatient Department Of A Tertiary Care Hospital Of Kolkata. Indian J Prev Soc Med. 2013;44:3-4.

8. Denny L. Cervical cancer: prevention and treatment. Discov Med. 2012;14:125-31.

9. Sankaranarayanan R, Budukh AM, Rajkumar R. Effective screening programs for cervical cancer in low and middle income developing countries. Bull World Health Organ. 2001;79:954-62.

10. Green JA, Kirwan JM, Tierney JF, Symonds P, Fresco L, Collingwood M, Williams CJ, et al. Survival and recurrence after concomitant chemotherapy and radiotherapy for cancer of the uterine cervix: a systematic review and metaanalysis. Lancet. 2001;358(9284):781-6.

11. Kirwan JM, Symonds P, Green JA, Tierney J, Collingwood M, Williams CJ. A systematic review of acute and late toxicity of concomitant chemoradiation for cervical cancer. Radiotherapy Oncol. 2003;68(3):217-26.

12. Le Borgne G, Mercier M, Woronoff AS, Guizard AV, Abeilard E, Caravati-Jouvenceaux A, et al. Quality of life in long-term cervical cancer survivors: a population-based study. Gynaecologic oncology. 2013;129(1):222-8.

13. Higham CE, Faithfull S. Bone health and pelvic radiotherapy. Clin Oncol. 2015;27(11):668-78.

14. Michaud LB, Goodin S. Cancer-treatment induced bone loss, part 1. Am J Health Syst Pharm. 2006;63:419-30.

15. Uezono H, Tsujino K, Moriki K, Nagano F, Ota Y, Sasaki R, et al. Pelvic insufficiency fracture after definitive radiotherapy for uterine cervical cancer: retrospective analysis of risk factors. J Radiat Res. 2013;54(6):1102-9.

16. Park SH, Kim JC, Lee JE, Park IK. Pelvic insufficiency fracture after radiotherapy in patients with cervical cancer in the era of PET/CT. Radiat Oncol J. 2011;29(4):269-76.

17. Baxter NN, Habermann EB, Tepper JE. Risk of pelvic fractures in older women following pelvic irradiation. JAMA. 2005;294:2587-93.

18. Pfeilschifter J, Diel IJ. Osteoporosis Due to Cancer Treatment: Pathogenesis and Management. J Clin Oncol. 2000;18:1570-93.

19. Nishio K, Tanabe A, Maruoka R, Nakamura K, Takai M, Sekijima T, et al. Bone mineral loss induced by anticancer treatment for gynecological malignancies in premenopausal women. Endocr Connect. 2012;2(1):11-7.

20. Oh YL, Yoon MS, Suh DS, Kim A, Kim MJ, Lee JY, et al. Changes in Bone Density after Cancer Treatment in Patients with Cervical and Endometrial Cancer. J Cancer. 2015;6(1):82-9.

21. Okonogi N, Saitoh JI, Suzuki Y, Noda SE, Ohno T, Oike $\mathrm{T}$, et al. Changes in bone mineral density in uterine cervical cancer patients after radiation therapy. Int $\mathrm{J}$ Radiat Oncol Biol Phys. 2013;87(5):968-74.

22. Kanis JA, Glüer CC. An update on the diagnosis and assessment of osteoporosis with densitometry Osteoporos Int. 2000;11(3):192-202.

23. Fairbank JCT, Couper J, Davies JB. The Oswestry Low Back Pain Questionnaire. Physiotherapy. 1980;66:271-3.

Cite this article as: Kumar D, Singh T, Bansal P, Rai B. A prospective study to evaluate pelvic bone mineral density changes in patients of cervical cancer undergoing chemoradiotherapy: study protocol. Int $\mathbf{J}$ Clin Trials 2019;6(4):215-8. 